\title{
Web and Social Media Searches Highlight Menstrual Irregularities as a Global Concern in COVID-19 Vaccinations
}

\section{Ariel Katz}

Ben-Gurion University of the Negev

\section{Yoav Tepper}

Ben-Gurion University of the Negev

Alal Eran ( $\sim$ alal_eran@hms.harvard.edu )

Ben-Gurion University of the Negev

Ohad Birk

Ben-Gurion University of the Negev

\section{Research Article}

Keywords: COVID-19 vaccination, menstrual cycle

Posted Date: February 25th, 2022

DOI: https://doi.org/10.21203/rs.3.rs-1338740/v1

License: (c) (i) This work is licensed under a Creative Commons Attribution 4.0 International License. Read Full License 


\section{Abstract \\ Background}

Delineation of public concerns that prevent vaccine compliance is a major step in generating assurances and enhancing the success of COVID-19 prevention programs. We therefore sought to identify public concerns associated with COVID-19 vaccines, as reflected by web and social media searches, with a focus on menstrual irregularities.

\section{Methods}

We used trajectory analyses of web and social media search data in combination with global COVID-19 data to reveal time-dependent correlations between vaccination rates and the relative volume of vaccine and period related searches.

\section{Results}

A surge of period and vaccine related google searches followed the commencement of vaccination programs in English speaking countries, and across the United States. TikTok users were more engaged in period problems in 2021 than ever before.

\section{Conclusions}

National and state-level correlations between COVID-19 vaccinations and online activity demonstrate a global major concern of vaccine-related menstrual irregularities. Whether it is a potential side effect or an unfounded worry, monitoring of web and social media activity could reveal the public perception of COVID-19 prevention efforts, which could then be directly addressed and translated into insightful public health strategies.

\section{Introduction}

Women around the world have reported a link between COVID-19 vaccinations and changes in their menstrual cycle regularity and intensity ${ }^{1}$. One of the early reports was a tweet by Kathryn Clancy, a Professor of Anthropology at the University of Illinois, which was followed by hundreds of responses of women identifying with her concerns, and in many cases reflecting on having similar menstrual irregularities following vaccination. Later reports on menstrual irregularities following vaccination were met with criticism² ${ }^{2}$, some suggesting that pandemic-related stress might be the cause of irregularities. Antivaxxers later highlighted the worries regarding possible vaccine-induced menstrual irregularities, expanding unverified and often manipulative concerns, and promoting fears of vaccine-associated 
abortions and infertility ${ }^{3,4}$. Recent studies have examined the effects of COVID-19 disease ${ }^{5}$ and vaccines $^{6,7}$ on menstrual irregularities, suggesting lack of adequate reporting of such irregularities due to women's reluctance to discuss these matters with their physicians ${ }^{8}$. Yet whether these are anecdotal reports amplified by antivaxxers or a true uncharted association remains largely unknown. To examine the spread and magnitude of this potential link we mined web and social media search data and its relation to the specific timing of national and state-level COVID-19 vaccination drives.

\section{Methods}

We used Google Trends ${ }^{9}$ to examine the relative volume of searches for the combination of "vaccine" and "period" in English speaking countries and across the US between January 2020 and November 2021. Google Trends, a powerful tool for analyzing population behavior and trends, has been utilized in several public health studies ${ }^{10}$, including in relation to the COVID-19 pandemic ${ }^{11,12}$. Google trends is capable of summarizing time and location-specific searches, enabling integrative spatiotemporal analyses.

We also mined TikTok, a social network serving more than a billion users, $53 \%$ of whom are female and $78 \%$ of whom are under the age of $24^{13}$. Using Analisa.io (https://analisa.io), we examined the activity of “\#periodproblems" from January 2019 to October 2021.

We integrated the search data with COVID-19 vaccination data obtained from Our World In Data (https://ourworldindata.org/covid-vaccinations) and other COVID-19 aggregated statistics, including the number of cases and deaths, obtained from https://github.com/CSSEGISandData/COVID-

19/tree/master/csse_covid_19_data. Our python code that performs and visualizes all analyses is freely available at https://github.com/SgtTepper/CovidSearchTrends/. Specifically, it mines Google Trends via pytrends, integrates the results with COVID statistics using pandas, computes Spearman correlations, plots co-occurring multimodal data on the same timeline using seaborn, and plots relative search volume by state using plotly.

\section{Results}

In English speaking countries, the relative volume of Google searches for the combination of terms "period" and "vaccine" grew dramatically following the initiation of COVID-19 vaccination drives, with a later 3-5-fold further increase once $\sim 50 \%$ of the population has been vaccinated (Fig. 1). Country-specific timing of surges in "vaccine and period" related searches and its temporal relation to the local vaccination drive control for possible effects of geographic location and the local culture. Note that the analyses focused on countries sharing English as the main language, ensuring the searches were done mostly with similar wording and adequate spelling.

Similar relations were detected within the United States (Fig. 2). Overall, Google searches for the combination of "period" and "vaccine" were frequent between October 2020 and October 2021, to a somewhat lesser extent in the mid-U.S (Fig. 2A). As exemplified in Fig. 2b-2m, searches for the combined 
terms "period" and "vaccine" became extremely prominent with the initiation of COVID-19 vaccines in each state. Notably, further major peaks in searches for the terms "period" and "vaccine" appeared 2-6 months following initiation of vaccines, suggesting that searches did not only reflect worries, but were possibly triggered by menstrual irregularities following vaccines.

Moreover, analysis of worldwide use and reactions to "\#periodproblems" in the video-focused social network TikTok between January 2019 and October 2021 (Fig. 3) demonstrated an initial significant peak of this hashtag in March 2020 (both in the number of exposures and the number of responses to the term), possibly reflecting pandemic stress. Yet this peak subsided and dramatically re-emerged in January 2021 with several major peaks, coinciding with the timing of initiation of vaccination programs around the world.

\section{Discussion}

This study finds a surge of Google searches related to vaccine and the menstrual cycle following the initiation of national and state-level COVID-19 vaccination drives. While it may suggest that COVID-19 vaccines could affect menstrual regularity, it could also reflect the mere concerns of those not yet vaccinated. We also find that TikTok hashtag \#periodproblems peaked at the beginning of the pandemic in March 2020, and again with the initiation of vaccination programs, early in 2021 . This reflects menstrual irregularities being either a consequence $s$ of the vaccine or a significant worry regarding such consequences, yet practically rules out claims that concerns about the menstrual cycle are merely an effect of pandemic-related stress.

The safety of COVID-19 vaccines in pregnancy and lack of discernable effects on fertility have been recently shown in several reports ${ }^{14,15}$. Studies of the possible effects of COVID-19 vaccines on menstruation have been initiated through questionnaires, doctors' reports and other experimental strategies ${ }^{16}$. However, these studies encompass limited amounts of data in comparison to that available by monitoring web and social media activity. Moreover, studies based on physicians' reports are lacking since women do not often report menstrual irregularities to their physicians, because of discomfort or inconvenience of going to the doctor, especially during the pandemic ${ }^{8}$. Our findings reflect menstrual cycle irregularities as a major worry of women in considering compliance with vaccination programs, suggesting possible effects of the vaccine on such irregularities, and highlight a clear need for scientifically sound studies testing possible effects of the vaccines on menstruation. Of note, our analysis deals with relative search volumes. Thus, significant changes in other searches at the same time and place might mask or distort the results.

This study highlights the use of an array of social media big data analyses, combining Google Trends and TikTok (representing a significantly younger cohort), in identifying public trends preventing vaccine compliance across different states and countries. Prior studies proved retrospectively through Google trends facts that were previously known, such as the initiation of the pandemic or effects of COVID-19 disease on smell. In contrast, this study highlights social media big data analysis as an effective 
massive-scale tool in the initial identification and determination of adverse effects (as well as positive effects) of the COVID-19 vaccine. Moreover, it verifies the findings across various states in the U.S. and other countries, ruling out effects of local trends, climates, publicities and regulations, as well as enables delineation of different effects of the various COVID-19 vaccines given in different states or countries. Thus, web and social media analyses enable improved strategies in identifying public health concerns and determining public health policies to improve vaccine compliance.

\section{Declarations}

\section{Data availability:}

All data and its visualizations generated as part of this study are available at https://bit.ly/covid_vaccine_period_search_data.

\section{References}

1. Male, V. Menstruation and covid-19 vaccination. BMJ 376, 0142 (2022).

2. Women Say COVID Vaccine Side Effects Impact Their Periods, So Why Don't Doctors Care? Haaretz.

3. Lu-Culligan, A. \& Iwasaki, A. Opinion I The False Rumors About Vaccines That Are Scaring Women. The New York Times (2021).

4. Butler, K. Social media influencers are spreading wild rumors about COVID-19 vaccines and periods. Mother Jones https://www.motherjones.com/politics/2021/04/social-media-influencers-arespreading-wild-rumors-about-covid-19-vaccines-and-periods/.

5. Li, K. et al. Analysis of sex hormones and menstruation in COVID-19 women of child-bearing age. Reprod Biomed Online 42, 260-267 (2021).

6. Alvergne, A. et al. COVID-19 vaccination and menstrual cycle changes: A United Kingdom (UK) retrospective case-control study. 2021.11.23.21266709 https://www.medrxiv.org/content/10.1101/2021.11.23.21266709v3 (2021) doi:10.1101/2021.11.23.21266709.

7. Edelman, A. et al. Association Between Menstrual Cycle Length and Coronavirus Disease 2019 (COVID-19) Vaccination: A U.S. Cohort. Obstetrics \& Gynecology 10.1097/AOG.0000000000004695 (2022) doi:10.1097/AOG.0000000000004695.

8. Seear, K. The etiquette of endometriosis: Stigmatisation, menstrual concealment and the diagnostic delay. Social Science \& Medicine 69, 1220-1227 (2009).

9. Google Trends. Google Trends https://trends.google.com/trends/explore.

10. Hartnett, K. P. et al. Syndromic Surveillance for E-Cigarette, or Vaping, Product Use-Associated Lung Injury. New England Journal of Medicine (2019) doi:10.1056/NEJMsr1915313.

11. Lu, T. \& Reis, B. Y. Internet search patterns reveal clinical course of COVID-19 disease progression and pandemic spread across 32 countries. npj Digit. Med. 4, 1-9 (2021). 
12. Mavragani, A. \& Gkillas, K. COVID-19 predictability in the United States using Google Trends time series. Sci Rep 10, 20693 (2020).

13. TikTok global creators by age 2021. Statista https://www.statista.com/statistics/1257721/tiktokcreators-by-age-worldwide/.

14. Male, V. Are COVID-19 vaccines safe in pregnancy? Nat Rev Immuno/ 21, 200-201 (2021).

15. Morris, R. S. SARS-CoV-2 spike protein seropositivity from vaccination or infection does not cause sterility. F\&S Reports 2, 253-255 (2021).

16. Petri, A. E. Researchers will study whether the vaccines affect women's periods. The New York Times (2021).

17. San Diego to take part in Moderna's massive COVID-19 vaccine trial - The San Diego Union-Tribune. https://www.sandiegouniontribune.com/business/biotech/story/2020-07-24/san-diego-to-take-partin-modernas-massive-covid-19-vaccine-trial.

18. Moderna's Fully Enrolled Phase 3 COVE Study of mRNA-1273 | Moderna, Inc. https://www.modernatx.com/cove-study.

19. Choi, K. R. A Nursing Researcher's Experience in a COVID-19 Vaccine Trial. JAMA Internal Medicine 181, 157-158 (2021).

\section{Figures}

\section{Figure 1}

Relative volume of Google searches for the combination of terms "period" and "vaccine", and their temporal relation to national vaccination rates in English speaking countries. (a) Australia, (b) Canada, (c) United Kingdom, (d) United States. The volume of "vaccine" searches is shown as a positive control.

\section{Figure 2}

State-level "vaccine and period" searches and their temporal relation to vaccination rates. (a) Relative volume of "vaccine and period" related searches across the United States between October $1^{\text {st }} 2020$ and October $1^{\text {st }}$ 2021. (b-m) Temporal relations of "vaccine and period" searches and state-specific vaccinations and deaths, normalized to their maxima within the studied period, namely 10/1/2020 10/1/2021 in (b) California, (c) Colorado, (d) Connecticut, (e) Illinois, (f) Kentucky, (g) Massachusetts, (h) Michigan, (i) Missouri, (j) New Hampshire, (k) New Mexico, (I) North Carolina, (m) South Carolina. Similar timelines for all 50 states are available at https://github.com/SgtTepper/CovidSearchTrends/. Of note, COVID-19 vaccinations started in December 2020 in California, while state-level vaccination statistics are available from January 2021. A possible explanation for the minor increase in search volume in 
November 2020 in California is the Phase III clinical trial of COVID-19 vaccine by both Pfizer and Moderna, which included thousands of California residents ${ }^{17-19}$.

\section{Figure 3}

Worldwide TikTok use of the hashtag \#periodproblems between January 2019 and October 2021. There is an initial dramatic peak of this hashtag (both in number of exposures to this term and number of responses to the term) in March 2020, possibly reflecting pandemic stress, yet this peak subsided and dramatically re-emerged as of January 2021 with several peaks, possibly reflecting timing of initiation of vaccines in different countries. Note the massive engagement in this topic during worldwide vaccination drives. (a) Exposures to TikTok videos containing "\#periodproblems". (b) Responses to TikTok videos containing "\#periodproblems". 